\title{
The Multi-Source Probabilistically Preemptive M/PH/1/1 Queue With Packet Errors
}

\author{
Ozancan Doğan ${ }^{(1)}$ and Nail Akar ${ }^{(\bullet)}$, Member, IEEE
}

\begin{abstract}
Analytical modeling of Age of Information (AoI) and Peak AoI (PAoI) has recently drawn a lot of attention in the context of quantitative assessment of information freshness in status update systems. In this paper, we study a probabilistically preemptive bufferless $\mathrm{M} / \mathrm{PH} / \mathbf{1} / \mathbf{1}$ queue fed with information update packets from $N$ separate information sources for which a new information packet arrival from source- $m$ is allowed to preempt a packet from source- $n$ in service, with a probability depending on $n$ and $m$. To make the model even more general than the existing ones, we assume a distinct phase-type (PHtype) service time distribution, a distinct packet error and retransmission probability, for each of the information sources. Using sample path arguments and the theory of Markov Fluid Queues (MFQ), the exact distributions of the AoI and PAoI are numerically obtained for each of the sources. Numerical examples are provided to demonstrate the impact of various system parameters on AoI performance. In the context of a two-source system, we present a methodology on how to optimally choose the preemption probabilities and packet generation rates so as to minimize certain AoI-oriented cost functions.
\end{abstract}

Index Terms-Age of information, peak age of information, PH-type distribution, preemptive queues, Markov fluid queues.

\section{INTRODUCTION}

$\mathbf{T}$ IMELY status updates are key for stable operation in networked control and monitoring systems. Lately, there has been substantial amount of interest centered around Age of Information (AoI) and Peak AoI (PAoI) processes in the fields of information theory and wireless communications in the context of quantitative assessment of information freshness in status update systems [1]-[8]. The survey [6] provides a relatively recent overview of the AoI concept and its applications. AoI performance-related studies include those that propose analytical models for AoI [9]-[11] and also research focusing on optimization of AoI-related performance metrics [7], [12]-[15]. In the general setting of the existing literature, the AoI metric keeps track of the staleness of a remote monitor's knowledge of a stochastic process randomly sampled and transmitted (in the form of information packets) by an

Manuscript received September 14, 2020; revised March 11, 2021 and June 29, 2021; accepted August 10, 2021. Date of publication August 20, 2021; date of current version November 18, 2021. Ozancan Doğan is supported in part by the $5 \mathrm{G}$ and Beyond Scholarship granted by the Information and Communication Technologies (ICTA) of Turkey and Vodafone Turkey. The associate editor coordinating the review of this article and approving it for publication was N. Pappas. (Corresponding author: Nail Akar.)

Ozancan Doğan is with Meteksan Defence Inc., 06800 Ankara, Turkey.

Nail Akar is with the Department of Electrical and Electronics Engineering, Bilkent University, 06800 Ankara, Turkey (e-mail: akar@ee.bilkent.edu.tr).

Color versions of one or more figures in this article are available at https://doi.org/10.1109/TCOMM.2021.3106347.

Digital Object Identifier 10.1109/TCOMM.2021.3106347 information source where the monitor and the source reside at two separate points in a lossy packet-based communications network. More formally, the AoI maintained at the monitor for a given source is defined as the time elapsed since the generation of the last successfully received update packet. Consequently, the AoI process turns out to be a cyclic process that increases in time with unit slope within a cycle with the exception that the AoI process undergoes abrupt downward jumps at status packet reception instances. After such a jump, a new cycle begins. Under continuous-time stationary scenarios, the AoI process is a stationary continuous-time, continuous-valued stochastic process for which our interest in this paper is in finding its exact steady-state distribution in a very general bufferless server setting. A related equally important process is the embedded discrete-time, continuous-valued PAoI process that is obtained by taking the peak values during each cycle of the AoI process [16], the exact steady-state distribution of which is also sought in this paper. We consider, in particular, the information update system presented in Fig. 1 comprising $N$ information sources each with a sensor along with a bufferless server and a (remote) monitor. The sensor associated with source- $n$ detects the changes of the source state and generates information packets that contain sensed data and a time stamp. The packet generation from source- $n$ is assumed to be Poisson with intensity $\lambda_{n}$ and packets are immediately passed on to the server. The server, on the other hand, is in charge of sending the information packets towards the remote monitor using a packet-switched communications network. An information packet is exposed to a random delay in the network which is referred to as the service time of a packet. Another assumption we make is that the monitor immediately sends back positive/negative acknowledgments to the server. Moreover, we assume a bufferless (no waiting room for new packets) preemption-capable server in this paper. In this setting, a new arriving information packet immediately starts to receive service if the server is idle, but is either discarded or allowed to preempt the packet in service if the server is busy upon arrival. To make the model general, we assume the following:

(i) A new information packet arrival from source- $m$ is allowed to preempt the packet in service from source- $n$ with probability depending on both $n$ and $m$ denoted by $P_{n, m}, m, n=1, \ldots, N$. The matrix $\boldsymbol{P}=\left\{P_{n, m}\right\}$ is called the preemption matrix of this system.

(ii) The service time of each source has a general phase-type (PH-type) distribution covering exponential, Hyperexponential, and Erlang distributions as subcases [17]. Moreover, 


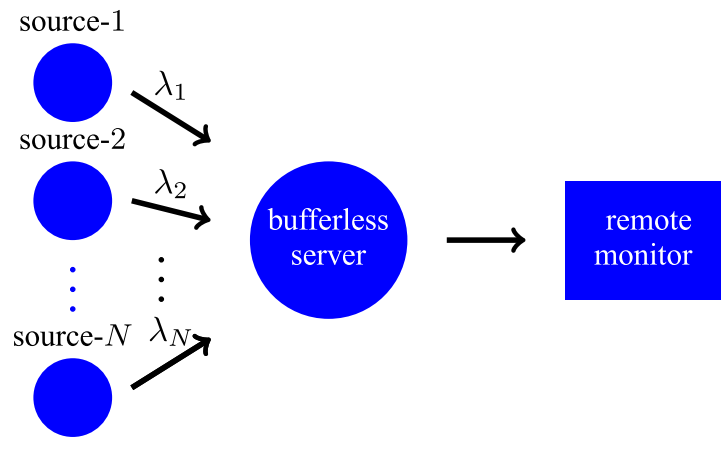

Fig. 1. $\quad N$ information sources transmitting status update packets to a remote monitor via a bufferless server.

the requirements of sources are heterogeneous, i.e., each source has its own service time requirement.

(iii) At the end of the service time needed for the delivery of an information packet, a packet error is said to occur with a probability depending on the source index. Upon a negative acknowledgment, this corrupt packet will be immediately retransmitted with a probability again depending on the source index, but is otherwise discarded. Therefore, when the server starts sending a packet, it stores a copy locally to potentially retransmit it. However, the system is still called bufferless since it does not provide a waiting room for new packets.

We call the above system a multi-source probabilistically preemptive bufferless M/PH/1/1 queue with packet errors, which is the focus of this paper. The AoI and PAoI processes will now be described for the bufferless server of interest by an illustrative example. Firstly, a successful information packet is defined as one which receives service until the end of its service time without being preempted and without a packet error whereas other information packets are deemed unsuccessful. Let $t_{j}^{(n)}$ represent the generation instance of the $j^{\text {th }}, j \geq 1$ successful source- $n$ information packet. Also let $u_{j}^{(n)}, j \geq 1$ denote the time at which the $j^{\text {th }}$ successful source$n$ packet is received by the monitor. $\Delta^{(n)}(t), t \geq 0$, denotes the continuous-time random process representing the AoI for source- $n$ at time $t$ with a given initial condition $\Delta^{(n)}(0)$. Note that this process has left-continuous sample paths as explained below. At $t=0, \Delta^{(n)}(t)$ increases linearly with time at a unit rate until the first successful packet is received at $t=u_{1}^{(n)}$. Subsequently, $\Delta^{(n)}\left(u_{1}^{(n)+}\right)$ is defined as $\lim _{t \downarrow u_{1}^{(n)}} \Delta^{(n)}(t)$ (the limit as $t$ approaches to $u_{1}^{(n)}$ by decreasing from the right or from above) which is set to $\Psi_{1}^{(n)}$ where $\Psi_{j}^{(n)}=u_{j}^{(n)}-t_{j}^{(n)}$ is the service time (or delay) of the $j^{\text {th }}$ successful source$n$ information packet. Then, the process $\Delta^{(n)}(t)$ increases with time in a similar way until the next successful class- $n$ packet reception. This pattern of increasing AoI and abrupt jumps at the reception instances is to repeat continuously. Let $\Phi_{j}^{(n)}=\Delta^{(n)}\left(u_{j}^{(n)}\right), j \geq 1$, denote the PAoI process for source- $n$. Actually, $\Phi_{j}^{(n)}$ is a discrete-time continuous-valued random process obtained by sampling the value of source$n$ AoI just before a successful source- $n$ packet reception. Let $\Delta^{(n)}, \Phi^{(n)}$, and $\Psi^{(n)}$ denote the steady-state values for the source- $n$ processes $\Delta^{(n)}(t), \Phi_{j}^{(n)}$, and $\Psi_{j}^{(n)}$, respectively. Moreover, we define the random variables $\Delta$ and $\Phi$ associated
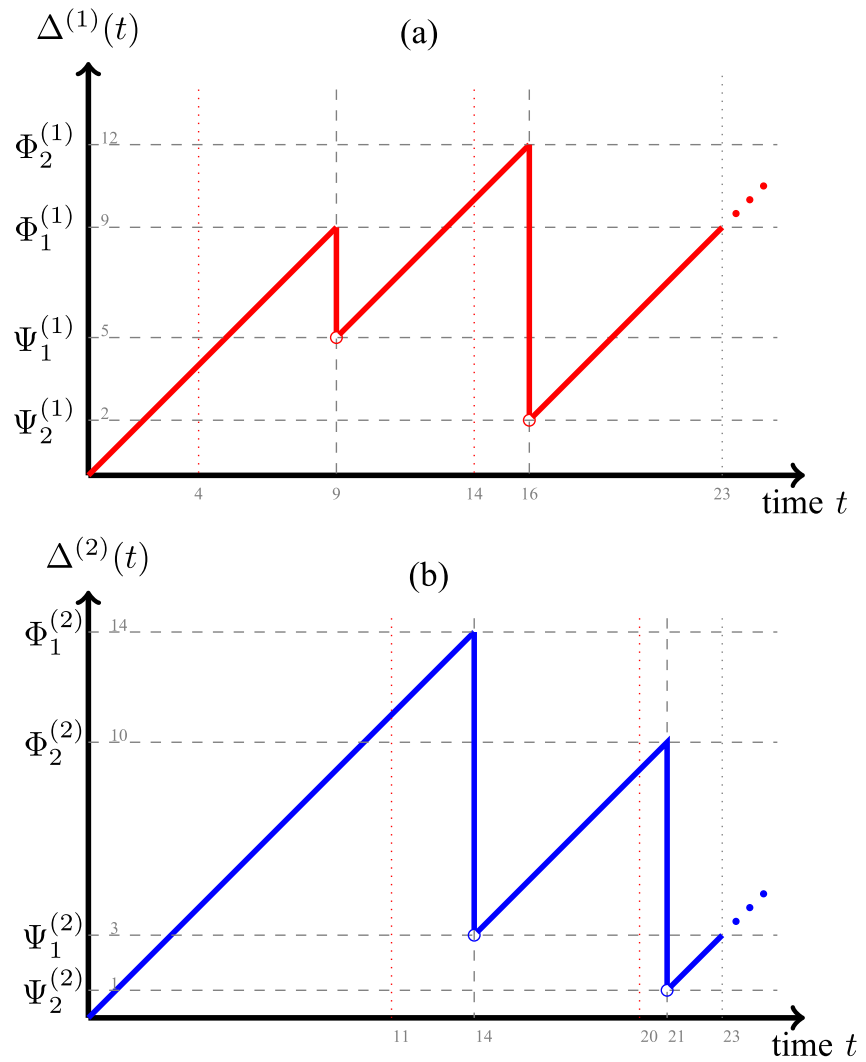

Fig. 2. Sample paths for the AoI processes (a) $\Delta^{(1)}(t)$ (b) $\Delta^{(2)}(t)$ for $0 \leq t \leq 23$ (both set to zero initially) for a two-source example scenario for which $\mathrm{P} i j$ denotes packet $-j$ arriving from source- $i$. P11 arrives at $t=1$ but is preempted by $\mathrm{P} 12$ at $t=4$ whose transmission is errored at $t=7$ which is then retransmitted and successfully received at $t=9$. P13 arrives at $t=10$ but is preempted by $\mathrm{P} 21$ at $t=11$ which is successfully received at $t=14$. P14 arrives at $t=14$ and is successfully received at $t=16$. On the other hand, P22 arrives at $t=16$ and is errored at $t=18$ and is not retransmitted. Finally, P23 arriving at $t=20$ is successfully received at $t=21$. The server is idle in the time intervals $(0,1),(9,10),(18,20)$, and $(21,23)$. The red dotted (gray dashed) vertical lines are for the successful packet arrival instances (reception instances).

with the system AoI and system PAoI, respectively, as follows:

$$
\Delta=\frac{1}{N} \sum_{n=1}^{N} \Delta^{(n)}, \quad \Phi=\frac{1}{N} \sum_{n=1}^{N} \Phi^{(n)},
$$

which are obtained by averaging the per-source steady-state AoI and PAoI variables, respectively. For the general $N$-source probabilistically preemptive $\mathrm{M} / \mathrm{PH} / 1 / 1$ system with packet errors (an illustration of which is given in Fig. 2 for $N=2$ ), we seek to find the following steady-state cdfs (cumulative distribution function) for the random variables $\Delta^{(n)}$ and $\Phi^{(n)}$, respectively:

$$
\begin{aligned}
& F_{\Delta^{(n)}}(x)=\lim _{t \rightarrow \infty} \operatorname{Pr}\left\{\Delta^{(n)}(t) \leq x\right\}, \\
& F_{\Phi^{(n)}}(x)=\lim _{j \rightarrow \infty} \operatorname{Pr}\left\{\Phi_{j}^{(n)} \leq x\right\} .
\end{aligned}
$$

Also let $f_{\Delta^{(n)}}(x)$ and $f_{\Phi^{(n)}}(x)$ for $x \geq 0$ represent the steady-state pdfs (probability density function) of the source$n$ AoI and PAoI processes, respectively.

Fig. 2 illustrates the sample paths of the AoI processes $\Delta^{(n)}(t), n=1,2$ for a two-source probabilistically preemptive bufferless server system with packet errors, starting from zero 
AoI values for each source at $t=0$. We conclude from Fig. 2 that each cycle of the AoI process $\Delta^{(n)}(t)$ consists of a linear part (with unit slope) beginning at the value $\Psi_{j}^{(n)}$ for some successful packet index $j$ for source- $n$ and evolving until the peak value $\Phi_{j+1}^{(n)}$. Actually, the sample path of the AoI process $\Delta^{(n)}(t)$ consists of an ordered concatenation of infinitely many linear parts (or called cycles). In order to numerically obtain the distribution of the AoI and PAoI processes, we propose to use the theory of Markov Fluid Queues (MFQ) [18]-[20]. The main idea is that we propose to construct a certain MFQ out of which sample paths can be generated whose certain parts overlap with the sample cycles of the AoI process for any source- $n$. Moreover, the sample paths generated by the constructed MFQ are to contain sample values that overlap with the sample values of the PAoI process and vice versa. Consequently, the cdfs of the AoI and PAoI processes given in (2) can be obtained out of the steady-state solution of the properly constructed MFQ. The main result of this paper is that, for each of the $N$ sources, the obtained cdfs are in matrix exponential form which are given below for a particular source of interest say source- 1 when $x \geq 0$ :

$$
\begin{aligned}
& F_{\Delta^{(1)}}(x)=1-\boldsymbol{g}_{\boldsymbol{A}} e^{\boldsymbol{A} x} \boldsymbol{h}_{\boldsymbol{A}}, \\
& F_{\Phi^{(1)}}(x)=1-\boldsymbol{g}_{\boldsymbol{P}} e^{\boldsymbol{A} x} \boldsymbol{h}_{\boldsymbol{P}},
\end{aligned}
$$

where the square matrix $\boldsymbol{A}$ is a stable matrix (or Hurwitz matrix), i.e., all its eigenvalues have strictly negative real parts. In Theorem 1, we will propose a method to obtain the matrix parameters of these two expressions, namely the parameters $\boldsymbol{A}, \boldsymbol{g}_{\boldsymbol{A}}, \boldsymbol{h}_{\boldsymbol{A}}, \boldsymbol{g}_{\boldsymbol{P}}$, and $\boldsymbol{h}_{\boldsymbol{P}}$, for the cdfs of the AoI and PAoI processes for source-1. Since the sources can always be renumbered, the form of (3) actually applies to each of the $N$ sources. However, the procedure detailed in Theorem 1 needs to be repeated for each source of interest to obtain the corresponding matrix parameters. The main contributions of this paper are the following:

(i) We present an analytical model for bufferless servers arising in status update systems that can be globally preemptive, self-preemptive, non-preemptive, etc. with a unifying probabilistic preemption framework. Besides unification, a probabilistic preemption policy different than the ones mentioned above can be optimum depending on the choice of system parameters and the cost function.

(ii) Packet errors and heterogeneous service time requirements across the sources makes the model even more general than the existing ones.

(iii) Most existing results use Stochastic Hybrid Systems (SHS) [8] while providing means to obtain the average AoI and PAoI values. The theory of SHS has also recently been used in [21] to obtain the moments and the moment generating functions (mgf) of the AoI process in certain scenarios. In this paper, we obtain the exact distributions of the AoI and PAoI processes in matrix exponential form given in Eqn. (3) using the alternative instrument of MFQs out of which the AoI and PAoI moments can very easily be obtained.

(iv) We use a similar MFQ-based methodology used in [22] for bufferless and single-buffer systems but extend its scope from a single-source setting to a multi-source system with heterogeneous service time requirements, probabilistic preemption, packet errors, and probabilistic retransmissions.

The organization of the paper is as follows. In Section II, related work is presented. Section III presents the notation throughout the paper as well as preliminaries on PH-type distributions and MFQs. Section IV presents the analytical model. In Section V, we provide numerical examples to validate the proposed approach and the impact of various system parameters with respect to certain AoI-related performance metrics. Finally, we conclude in Section VI.

\section{RELATED WORK}

The AoI concept was first introduced in [2] in the context of a single-source, single-server M/M/1 queueing model. This model is then extended to multiple sources in [23] and since then the single-server model in Fig. 1 for AoI has extensively been used for status update system modeling in the literature [2], [6]. There are many variations of this single-server queueing system studied in the recent literature depending on

- the number of sources (single vs. multiple) feeding the queue with information packets,

- the existence of errors during transmission, and retransmission policies,

- the generality of the distributions assumed for the inter-arrival times and service times,

- the queue capacity that places a limit on the number of packets that can be accommodated by the queueing system (either in service or in the waiting room),

- the scheduling discipline to be used whether it be First Come First Serve (FCFS), Preemptive Last Come First Serve (P-LCFS), Non-preemptive LCFS (NP-LCFS), etc.

- the buffer management scheme that is in charge of packet dropping at the server,

- the performance metric of interest (average AoI and PAoI values vs. their exact distributions).

Related work on AoI analytical models is briefed first in the single-source setting and subsequently for the multi-source scenario. In [2], the mean AoI is obtained for the single-source $\mathrm{M} / \mathrm{M} / 1, \mathrm{M} / \mathrm{D} / 1$, and $\mathrm{D} / \mathrm{M} / 1$ queues with infinite buffer capacity and FCFS scheduling. In [24], expressions are derived for the LST transform of the stationary distributions of the AoI and the PAoI processes in M/GI/1 and GI/M/1 queues. Despite the fact that relatively large buffers and FCFS scheduling are the de-facto choices in operational packet-switched communication networks, such choices have been shown to give rise to poor AoI performance in moderate to high load regimes. The reference [10] studies the AoI and PAoI distributions for small buffer systems, including the conventional M/M/1/1 and $\mathrm{M} / \mathrm{M} / 1 / 2$ queues, as well as the so-called $\mathrm{M} / \mathrm{M} / 1 / 2^{*}$ queue, for which the packet waiting in the queue is to be replaced by a newer packet arrival, which actually is a non-preemptive LCFS system. The mean AoI and PAoI figures in the preemptive LCFS M/G/1/1 queueing system is studied in [25] where a new arrival preempts the packet in service and the service time distribution is assumed to follow a more general gamma distribution. Exact PAoI expressions are derived in an $\mathrm{M} / \mathrm{M} / 1$ queueing system with packet delivery errors using 
different scheduling policies such as FCFS, P-LCFS, and NP-LCFS in [11]. The reference [9] provides expressions for the steady-state distributions of AoI and PAoI for a general class of single-source systems. A recent work in [22] also obtains the exact distributions of AoI and PAoI in bufferless systems with probabilistic preemption while allowing $\mathrm{PH}$-type distributions for both interarrival and service times. A similar model is also proposed in [22] for a single-buffer system with Poisson packet arrivals and PH-distributed service times allowing probabilistic replacement of the waiting packet by a newer packet arrival. A discrete-time queueing model with Bernouilli arrivals and geometric service times, using FCFS and non-preemptive LCFS scheduling is presented in [26] with expressions for the mean AoI and PAoI values.

In the multi-source setting, the reference [12] derives the mean PAoI expression for $\mathrm{M} / \mathrm{G} / 1$ and $\mathrm{M} / \mathrm{G} / 1 / 1$ systems with heterogeneous service time requirements which enables one to optimize system cost, as a function of mean PAoI, by choice of the update interval. The authors of [8] study the multi-source $\mathrm{M} / \mathrm{M} / 1$ model with FCFS as well as two variations (preemptive and non-preemptive with replacement) of LCFS using the theory of SHS and obtain exact expressions for the mean AoI. A preemptive $M / G / 1 / 1$ queue is considered in [27] with a common service time for all sources in which expressions for the mean AoI and PAoI are derived. A similar preemptive $\mathrm{M} / \mathrm{G} / 1 / 1$ system is studied in [28] allowing packet delivery errors. The authors [28] allow preemption of a source in service by a newly arriving packet from the same source and derive the mean AoI expressions for each source using the SHS technique. The reference [29] considers a two-source $\mathrm{M} / \mathrm{M} / 1 / 2$ queueing system in which a packet waiting in the queue can be replaced only by a newly arriving packet from the same source, again using SHS techniques. A non-preemptive $\mathrm{M} / \mathrm{M} / 1 / \mathrm{m}$ with common service times across sources is again studied by the SHS technique in [30] and mean AoI expressions are derived. A more general Hyperexponential $\left(\mathrm{H}_{2}\right)$ service time distribution for each class is considered in [31] for an $\mathrm{M} / \mathrm{H}_{2} / 1 / 1$ non-preemptive bufferless queue to derive an expression for the mean AoI per class. The authors of [32] derive an exact expression for the average AoI for the multi-source $\mathrm{M} / \mathrm{M} / 1$ FCFS queue and propose various approximate expressions for the average AoI for a multi-source M/G/1 FCFS queue. Recently, the mgf of AoI has been studied in [21] whereas a similar work derives the mgf of the AoI of each source in a bufferless multi-source status update system using global preemption [33]. Another recent work studies non-preemptive bufferless, globally preemptive bufferless, and single buffer with replacement policies in discrete-time for a multi-source status update system and derives the exact distributions of AoI and PAoI [34].

\section{PRELIMINARIES}

We use uppercase bold letters for denoting real-valued matrices and lowercase bold letters or symbols for vectors. $A_{i, j}$ denotes the $(i, j)$ th entry of a matrix $\boldsymbol{A}$ whereas the $j$ th entry of a row or column vector $\boldsymbol{\alpha}$ is $\alpha_{j}$. The notations $\mathbf{0}_{k \times \ell}$, $\boldsymbol{I}_{m}$, and $\mathbf{1}_{n}$ are used to denote the matrix of zeros of size $k \times l$, identity matrix of size $m$, and a column matrix of ones of size $n$, respectively. When used without a subscript, it is left to the reader to infer the size information from the context. The notation $\boldsymbol{A} \otimes \boldsymbol{B}$ denotes the Kronecker product of the matrices $\boldsymbol{A}$ and $\boldsymbol{B}$. The notation $[\boldsymbol{\alpha}, \boldsymbol{\beta}]$ stands for the concatenation of the two row vectors $\boldsymbol{\alpha}$ and $\boldsymbol{\beta}$. The unit step function (or the Heaviside step function) is denoted by $u(x)$ which is one when $x \geq 0$ and zero elsewhere. The Dirac delta function (or the unit impulse function) is denoted by $\delta(x)$.

\section{A. Phase-Type Distributions}

Phase-type (PH-type) distributions have been instrumental for modeling independent and identically distributed (iid) non-exponential interarrival and/or service times in queueing systems [17]. This stems from the denseness property of PH-type distributions in the field of positive-valued distributions [35] as a result of which any positive-valued distribution can appropriately be approximated by $\mathrm{PH}$-type distributions; see [36] and [37] for two representative approximation algorithms. Moreover, finding the steady-state and transient solutions of queueing systems involving PH-type distributions is algorithmically possible as in the case of exponentially distributed interarrival and service times; see [17] and the references therein. We define a Markov process with the statespace $\mathcal{S}=\{1,2, \ldots, m, m+1\}$ with $m$ transient states, one absorbing state $m+1$, initial probability vector $\left[\boldsymbol{\sigma}, \sigma_{0}\right]$, and an infinitesimal generator

$$
\left[\begin{array}{ll}
S & \nu \\
0 & 0
\end{array}\right]
$$

where $\boldsymbol{\sigma}$ is a row vector of size $m, \sigma_{0}=1-\boldsymbol{\sigma} \mathbf{1}$, the subgenerator $\boldsymbol{S}$ is $m \times m$, and $\boldsymbol{\nu}$ is a column vector of size $m$ such that $\boldsymbol{\nu}=-\boldsymbol{S 1}$. The time required until absorption to the state $m+1$, denoted by $X$, is said to be of PH-type of order $m$ which is characterized with the matrix pair $(\sigma, S)$, i.e., $X \sim$ $P H(\sigma, S)$. In the majority of the research studies including the current paper, the probability mass at zero denoted by $\sigma_{0}$ is zero in which case $\sigma$ is called the initial probability vector. The cdf and the pdf of $X \sim P H(\sigma, S)$, denoted by $F_{X}(x)$ and $f_{X}(x)$, respectively, are given as:

$$
\begin{aligned}
F_{X}(x) & =\left(1-\boldsymbol{\sigma} e^{\boldsymbol{S} x} \mathbf{1}\right) u(x), \\
f_{X}(x) & =\boldsymbol{\sigma} e^{\boldsymbol{S} x} \boldsymbol{\nu} u(x)+\sigma_{0} \delta(x) .
\end{aligned}
$$

The following are the special cases of PH-type distributions: Exponential distribution with one transient state, Erlang distribution with 2 or more identical transient states visited in sequence, Hypoexponential distribution with 2 or more transient states visited in sequence which can be non-identical, Coxian distribution with 2 or more (not necessarily identical) transient states visited in sequence with a probability of transitioning to the absorbing state after each state, Hyperexponential distribution being a mixture of 2 or more exponential distributions. However, PH-type distributions generalize all these well-known distributions by allowing arbitrary transition patterns among the transient states and from the transient states to the absorbing state. Moreover, there are well-established algorithms for constructing PH-type random variables with 
general transition patterns whose distributions match with empirical distributions obtained from data or with a given pdf; see for example the Expectation-Maximization (EM) algorithm of [36] and the moment-matching algorithm of [37].

\section{B. Markov Fluid Queues}

We define a joint Markovian process, i.e., MFQ process, denoted by $\boldsymbol{X}(\boldsymbol{t})=\left(X_{f}(t), X_{m}(t)\right), t \geq 0$, where $0 \leq$ $X_{f}(t)<\infty$ is the continuous-valued fluid level in the buffer and the modulating process $X_{m}(t) \in \mathcal{S}=\{1,2, \ldots, n\}$ is a finite Continuous Time Markov Chain (CTMC) with state space $\mathcal{S}$ whose cardinality $n$ stands for the size of the MFQ. When $X_{f}(t)>0, X_{m}(t)$ is governed by generator $\boldsymbol{Q}$ whereas it is governed by $\tilde{Q}$ not necessarily the same as $\boldsymbol{Q}$ when $X_{f}(t)=0$. The drift of the fluid level is denoted by $r_{i}$ when $X_{m}(t)$ resides at state $i$ and $\boldsymbol{R}$ denotes the diagonal matrix of drifts, i.e., $\boldsymbol{R}=\operatorname{diag}\left\{r_{1}, r_{2}, \ldots, r_{n}\right\}$. We assume there are $b$ states with positive drift and $a=n-b$ states with negative drift. We do not envision zero drift states in this paper. When $X_{f}(t)=0$ and $X_{m}(t)=i$ with $r_{i}<0$, $X_{f}(t)$ is assumed to stay at zero since the fluid level cannot be negative. The matrix triple $(\boldsymbol{Q}, \tilde{\boldsymbol{Q}}, \boldsymbol{R})$ is said to entirely characterize the MFQ process $\boldsymbol{X}(\boldsymbol{t})$ or equivalently we say $\boldsymbol{X}(\boldsymbol{t}) \sim \operatorname{MFQ}(\boldsymbol{Q}, \tilde{\boldsymbol{Q}}, \boldsymbol{R})$. Steady-state distribution of the joint process $\boldsymbol{X}(t)$ is derived in [20] and [18] by using the eigendecomposition of a matrix of size $n$ for the case $Q=\tilde{Q}$. The steady-state joint cdf vector and the joint pdf vector of the MFQ $\boldsymbol{X}(t)$ (for $x \geq 0$ ) defined by

$$
\begin{aligned}
\boldsymbol{F}(\boldsymbol{x}) & =\left[F_{1}(x), F_{2}(x), \ldots, F_{n}(x)\right], \\
F_{i}(x) & =\lim _{t \rightarrow \infty} \operatorname{Pr}\left\{X_{f}(t) \leq x, X_{m}(t)=i\right\}, \\
\boldsymbol{f}(\boldsymbol{x}) & =\left[f_{1}(x), f_{2}(x), \ldots, f_{n}(x)\right], \quad f_{i}(x)=\frac{\mathrm{d}}{\mathrm{d} x} F_{i}(x),
\end{aligned}
$$

completely describe the steady-state behavior of the MFQ $\boldsymbol{X}(\boldsymbol{t})$ for which we have proposed in [22] a numerical algorithm for the scenario $Q \neq \tilde{Q}$ which turns out to be a special case of the more general multi-regime MFQs with well-established numerically efficient steady-state solvers [38]-[40]. Moreover, in the special case of only one state with negative drift, i.e., $a=1(b=n-1$ states with positive drift), a case that also arises in the current paper, a more specific method is given in [22, Algorithm 1] that relies on only basic matrix operations by which one can obtain the following matrix exponential expression for the steady-state joint pdf and cdf vectors:

$$
\begin{aligned}
& \boldsymbol{f ( x )}=\boldsymbol{v} e^{\boldsymbol{A} x} \boldsymbol{H} u(x)+\boldsymbol{c} \delta(x), \\
& \boldsymbol{F}(\boldsymbol{x})=\int_{0^{-}}^{x} \boldsymbol{f}(\boldsymbol{\tau}) \mathrm{d} \tau=\left(\boldsymbol{g} e^{\boldsymbol{A} x} \boldsymbol{H}+\boldsymbol{d}\right) u(x),
\end{aligned}
$$

where $\boldsymbol{v}, \boldsymbol{A}, \boldsymbol{H}, \boldsymbol{c}$ are $1 \times b, b \times b, b \times n$, and $1 \times n$, respectively, $\boldsymbol{A}$ is a stable matrix and

$$
g=v A^{-1}, d=c-v A^{-1} H .
$$

Note that $f_{i}(x)=\boldsymbol{v} e^{\boldsymbol{A} x} \boldsymbol{h}_{i} u(x)+c_{i} \delta(x)$ and $F_{i}(x)=$ $\left(\boldsymbol{g} e^{\boldsymbol{A} x} \boldsymbol{h}_{i}+d_{i}\right) u(x)$ with $\boldsymbol{h}_{\boldsymbol{i}}$ denoting the $i$ th column of $\boldsymbol{H}$.

\section{Analytical Model for the Multi-Source Preemptive M/PH/1/1 Queue}

We consider the status update system in Fig. 1 with $N$ sources, a server, and a monitor. The source $n, n=1, \ldots, N$ generates packets that carry status update information, according to a Poisson process with intensity $\lambda_{n}$. The traffic intensity vector is denoted by $\boldsymbol{\lambda}=\left[\lambda_{1}, \ldots, \lambda_{N}\right]$. A source- $n$ information packet immediately starts to receive service from the server when it finds the server idle upon arrival and its service time $\Theta^{(n)} \sim P H\left(\sigma^{(n)}, S^{(n)}\right)$ with order $\ell_{n}, \nu^{(n)}=$ $-\boldsymbol{S}^{(n)} \mathbf{1}, \boldsymbol{\sigma}^{(n)} \mathbf{1}=1$, and $E\left[\Theta^{(n)}\right]=\frac{1}{\mu_{n}}$ with $\mu_{n}$ denoting the average service rate of source- $n$. Let the per-source load be defined as $\rho_{n}=\frac{\lambda_{n}}{\mu_{n}}$ and the total load $\rho=\sum_{n=1}^{N} \rho_{n}$. We define the total order $\ell_{T}=\sum_{n=1}^{N} \ell_{n}$. We are given a preemption matrix $\boldsymbol{P}$ so that while the source- $n$ information packet is in service, a new information packet arrival from source $m, m=1, \ldots, N$ is allowed to preempt the packet in service with probability $P_{n, m}$. For each source $n$, we define the total intensity of traffic that can preempt a source- $n$ packet in service as $\bar{\lambda}_{n}=\sum_{m=1}^{N} \lambda_{m} P_{n, m}$. The following sub-cases of a general preemption matrix $\boldsymbol{P}$ have been studied in the literature:

(a) $\boldsymbol{P}=\mathbf{0}$ refers to a non-preemptive (NP) system [6] for which preemption is not allowed at all.

(b) $\boldsymbol{P}=\mathbf{1}_{N} \mathbf{1}_{N}^{T}$ stands for global preemption (GP) in [8] for which a fresh (newly arriving) source- $m$ packet is allowed to preempt any source- $n$ packet in service for $n=1, \ldots, N$ including a packet in service from the same source.

(c) $\boldsymbol{P}=\boldsymbol{I}_{\boldsymbol{N}}$ represents a self-preemptive (SP) system in [28] for which a fresh source- $m$ packet is only allowed to preempt a packet from source- $m$ in service.

(d) $\boldsymbol{P}$ being lower triangular with all non-zero entries set to one representing prioritized preemption (PP) when a source$n$ packet in service is to be preempted by a fresh source- $m$ packet as long as $m \leq n$ in which case source-1 (source- $N$ ) is said to have the highest (lowest) priority.

In this paper, we propose a model with general preemption matrix $\boldsymbol{P}$ that covers all the sub-cases described above. In all such systems, preemption may potentially be beneficial for two different reasons. Firstly, a new information packet always carries more timely information than the one in service. Secondly, sources can be differentiated from each other by proper choice of preemption probabilities.

In our proposed model, we also consider error-prone channels. At the end of the service time of a source- $n$ packet, a transmission error is detected at the monitor with probability $e_{n}$. The successful transmission probability of a source- $n$ packet is denoted by $q_{n}=1-e_{n}$. An errored class- $n$ packet is retransmitted with probability $r_{n}$ irrespective of how many times it was transmitted. We let $d_{n}=1-r_{n}$ denote the discarding probability of an errored class- $n$ packet. For the sake of convenience, the service times of fresh information packets and retransmitted packets are assumed to be independent. Recall that an information packet which is preempted by another packet or discarded after a number of retransmissions, is an unsuccessful packet. Successful packets are those that are neither preempted nor discarded. We tag the specific source-1 for which the exact distributions of the AoI and PAoI 
TABLE I

Description of The Four Subsets of States For the MFQ $\boldsymbol{X}(t)$

\begin{tabular}{|l|l|l|}
\hline Subset & State & Description \\
\hline $\mathcal{S}_{1}$ & $(1, j)$ & $\begin{array}{l}\text { The first source-1 packet is in service, the service } \\
\text { state is } j, 1 \leq j \leq \ell_{1} \text {, and the fluid level increases } \\
\text { with unit rate. }\end{array}$ \\
\hline $\mathcal{S}_{2}$ & 2 & $\begin{array}{l}\text { The server is idle, we wait for a new packet arrival, } \\
\text { and the fluid level increases with unit rate. }\end{array}$ \\
\hline $\mathcal{S}_{3}$ & $(3, n, j)$ & $\begin{array}{l}\text { The server is busy serving a source- } n \text { packet, } 1 \leq \\
n \leq N, \text { the service state is } j, 1 \leq j \leq \ell_{n}, \text { and the } \\
\text { fluid level increases with unit rate. When } n=1, \\
\text { the information packet in service is subsequent to } \\
\text { the first source-1 packet described in subset } \mathcal{S}_{1} .\end{array}$ \\
\hline $\mathcal{S}_{4}$ & 4 & $\begin{array}{l}\text { The fluid level decreases with unit rate to prepare } \\
\text { for the next cycle. When the zero fluid level is hit, } \\
\text { a transition to state }(1, j) \text { occurs with intensity } \\
\sigma_{j}^{(1)} \text { according to the service time distribution of } \\
\text { source-1 packets. }\end{array}$ \\
\hline
\end{tabular}

processes are to be obtained but if the interest is on another information source- $n$ where $n \neq 1$, the same procedure can be repeated by renumbering the sources. For this purpose, we construct an MFQ process $\boldsymbol{X}(t)=\left(X_{f}(t), X_{m}(t)\right)$ with $X_{m}(t) \in \mathcal{S}=\cup_{i=1}^{4} \mathcal{S}_{i}$, where each subset of states is described in Table I. This MFQ generates a fluid level trajectory comprising infinitely many cycles where cycle- $k, k=1,2, \ldots$, is initiated with the arrival of a source-1 information packet that is accepted by the server into service leading to a visit of some state $(1, j)$ in $\mathcal{S}_{1}$ according to the initial probabilities $\sigma_{j}^{(1)}$ at instant $\tau_{k}$ when the fluid level is zero. Note that $\sigma^{(1)}$ is the initial probability vector of $\Theta^{(1)}$. Subsequently, the fluid level trajectory keeps track of time elapsed from the time instant $\tau_{k}$ described as follows. When this source-1 packet is preempted or discarded due to errors, then the packet is unsuccessful and the corresponding cycle is an unsuccessful cycle in which case we transition to the single state 4 in $\mathcal{S}_{4}$ upon preemption or discarding in order to prepare for the next cycle. Since a cycle is to start at a zero fluid level, in state 4, the fluid level decreases at a unit rate until the zero fluid level is hit. If the first source-1 packet is successfully transmitted in the first transmission, or in one of the next retransmission attempts, then the packet is successful and the corresponding cycle is a successful cycle in which case the fluid level trajectory will keep track of time elapsed from the instant $\tau_{k}$ until the reception of the next successful source-1 packet. Subsequently, once the next source-1 packet reception occurs, we transition to state 4 in $\mathcal{S}_{4}$ to terminate the current successful cycle and prepare for the next cycle. Fig. 3(a) provides a sample path of the fluid level process $X_{f}(t)$. In cycle-1, the first source-1 packet is preempted and is therefore unsuccessful. In cycle-2, the first source-1 packet is not preempted but is discarded. Therefore, cycle-2 is also an unsuccessful cycle. In cycle- 3 , the first source-1 packet (arriving at system at $\tau_{3}$ ) transmission is successfully over (shown by the end of blue dashed curve) after which the server is idle in state 2 in $\mathcal{S}_{2}$. With a new source- $n$ arrival, we transition to a state $(3, n, j) \in \mathcal{S}_{3}$. In the case of a successful transmission of source- $n$ packets for $n \neq 1$ or any source- $n$ packet discarding, we transition back to state 2 . In the case of preemption, we stay at $\mathcal{S}_{3}$. We transition to state 4 when a successful transmission of source-1 packet occurs from the state $(3,1, j)$ for some service state $j, 1 \leq j \leq \ell_{1}$. Note that the fluid level at the end of $\mathcal{S}_{1}$ in successful cycles has the same distribution as $\Psi^{(1)}$ since the latter is the distribution of the service time of successful source-1 packets. Moreover, the fluid level at the transition instance from $\mathcal{S}_{3}$ to state 4 has the same distribution as $\Phi^{(1)}$. A sample cycle of the AoI process for source-1 is depicted in Fig. 3(b) which starts from a value distributed according to $\Psi^{(1)}$ and linearly increases until the value $\Phi^{(1)}$. Therefore, we conclude that part of the fluid level process in a successful cycle of the MFQ $\boldsymbol{X}(t)$ spent in states of $\mathcal{S}_{2}$ and $\mathcal{S}_{3}$ overlaps with one cycle of the AoI process.

Let us now rigorously describe the operation of the MFQ $\boldsymbol{X}(\boldsymbol{t})$. A cycle of the fluid level process $X_{f}(t)$ begins with a visit to a state in $\mathcal{S}_{1}$ when the fluid level is zero. Let us assume that we are currently at state $(1, j), 1 \leq j \leq \ell_{1}$, from which a transition to state $(1, k), k \neq j$ occurs with intensity $S_{j, k}^{(1)}$ when the service state changes, or to state $(1, k)$ with intensity $\nu_{j}^{(1)} e_{1} r_{1} \sigma_{k}^{(1)}$ when the service time of the first source-1 packet is over, but it is errored and retransmitted. A transition to state 4 occurs with intensity $\bar{\lambda}_{1}$ when the source-1 packet is preempted by a new arrival or with intensity $\nu_{j}^{(1)} e_{1} d_{1}$ when the service time of the packet is over but the packet is errored and discarded. Finally, we have a transition to state 2 with intensity $\nu_{j}^{(1)} q_{1}$ when the service time of the packet is successfully over. With the transitions described above, we ensure that when we are at the beginning of state 2 , the fluid level has risen to a level distributed according to $\Psi^{(1)}$. When in state 2 , a transition to state $(3, n, j)$ occurs with intensity $\lambda_{n} \sigma_{j}^{(n)}$. Let us now assume that we are at state $(3, n, j)$ in $\mathcal{S}_{3}$ during which the fluid level continues to rise again with unit slope. Therefore, a source- $n$ packet is in service and we are in service state $j$. We have the following transition possibilities from state $(3, n, j)$. For $k \neq j$, a transition to state $(3, n, k)$ occurs with intensity $S_{j, k}^{(n)}$ whereas a transition to state $(3, n, k)$ is incurred with intensity $\nu_{j}^{(n)} e_{n} r_{n} \sigma_{k}^{(n)}$ when the service time of the source- $n$ packet is over but it is errored and retransmitted. We transition to state $(3, m, l)$ with intensity $P_{n, m} \lambda_{m} \sigma_{l}^{(m)}$ when the source- $n$ packet is preempted by a new information packet from source- $m$ whereas we transition to state 2 with intensity $\nu_{j}^{(n)} e_{n} d_{n}$ when the service time of the source- $n$ packet is over but the packet is errored and discarded. For $n \neq 1$, a transition to the same state 2 occurs with intensity $\nu_{j}^{(n)} q_{n}$ when the service time of the source- $n$ packet is successfully over. On the other hand, when $n=1$, we transition to state 4 with intensity $\nu_{j}^{(1)} q_{1}$; see the transition instance from $\mathcal{S}_{3}$ to $\mathcal{S}_{4}$ in cycle-3 of Fig. 3. When at state 4 , the fluid level decreases with a unit rate without any state changes until the fluid level zero is hit. The only way to escape from this particular state is through a transition to state $(1, j)$ with intensity $\sigma_{j}^{(1)}$. These transition instances coincide with the instances $\tau_{k}, k=1,2, \ldots$, in Fig. 3 at which cycle$k$ is initiated and it is clear that a new cycle starts at state $(1, j)$ with probability $\sigma_{j}^{(1)}$. We have the following detailed observations about the proposed MFQ model: 


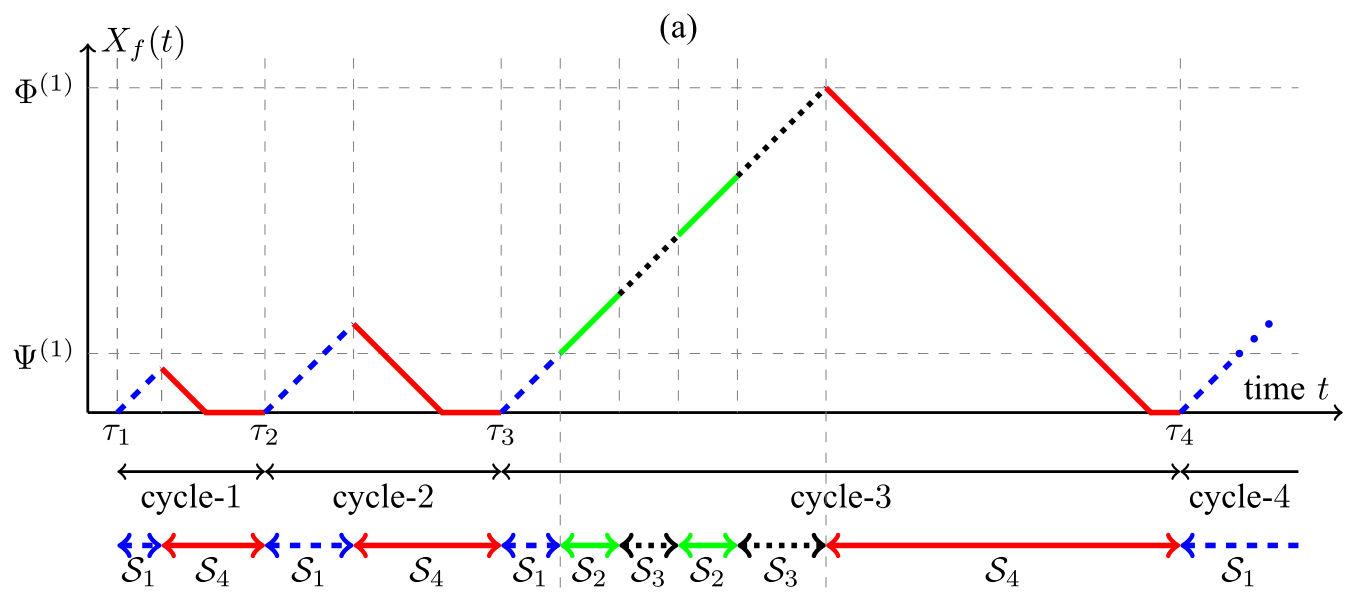

(b)

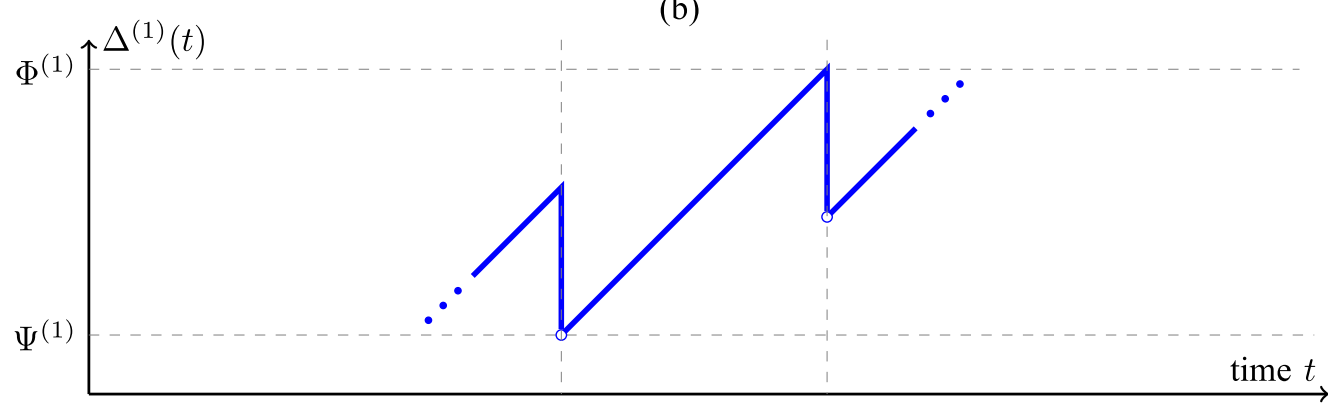

Fig. 3. (a) Sample path of the fluid level process $X_{f}(t)$ (b) Sample path of the AoI process $\Delta^{(1)}(t)$. The first two cycles of $X_{f}(t)$ are unsuccessful cycles. Part of the successful cycle-3 spent in states of $\mathcal{S}_{2}$ and $\mathcal{S}_{3}$ overlaps with one cycle of the AoI process.

(a) The transition from the subset $\mathcal{S}_{2}$ to $\mathcal{S}_{3}$ occurs twice in cycle-3 for the example of Fig. 3 before a transition takes place to state 4 . However, in general, there is no limit on how many times a transition occurs from subset $\mathcal{S}_{2}$ to $\mathcal{S}_{3}$ within a successful cycle.

(b) Part of the successful cycles (corresponding to subsets $\mathcal{S}_{2}$ and $\mathcal{S}_{3}$ ) of the proposed MFQ coincide with the individual cycles of the AoI process in no particular order. Therefore, the cdfs of the steady-state AoI and PAoI processes can be shown to be obtained from the joint steady-state cdf vector of the proposed MFQ (see the constructive proof of Theorem 1). However, a sample path of the fluid level process of our proposed MFQ does not coincide with the sample path of the AoI process. Consequently, our proposed MFQ does not model the dependence between consecutive AoI cycles. However, this does not provide any inconvenience as far as the steady-state AoI and PAoI distributions are sought.

(c) The particular state 4 is an auxiliary state used for terminating either a successful or unsuccessful MFQ cycle and also initiating a new MFQ cycle from the right initial condition. Therefore, the time spent in $\mathcal{S}_{4}$ does not play any role on the sample paths of the actual AoI process. Similarly, the particular states $(1, j)$ in $\mathcal{S}_{1}$ are also auxiliary states and they are constructed in such a way that when we make a transition from $\mathcal{S}_{1}$ to $\mathcal{S}_{2}$, the fluid level is distributed according to $\Psi^{(1)}$.

With the lexicographical ordering of the states from subset $\mathcal{S}_{1}$ to $\mathcal{S}_{4}$ and rewriting the transitions in matrix form, we obtain $\boldsymbol{X}(\boldsymbol{t}) \sim \operatorname{MFQ}(\boldsymbol{Q}, \tilde{\boldsymbol{Q}}, \boldsymbol{R})$ with size $\ell=\ell_{T}+\ell_{1}+2$ where $\boldsymbol{Q}$ is given as in Eqn. (11), as shown at the bottom of the next page, where

$$
V^{(j)}=\nu^{(j)} \otimes \sigma^{(j)}, \quad G^{(j)}=1 \otimes \sigma^{(j)},
$$

and the matrix $\tilde{Q}$ is the same as $Q$ except for the block entry in the south-west corner which is set to $\boldsymbol{\sigma}^{(1)}$, and for the scalar at the south-east corner which is set to -1 . Moreover,

$$
\boldsymbol{R}=\operatorname{diag}\left\{\boldsymbol{I}_{\ell_{T}+\ell_{1}+1},-1\right\} .
$$

There are $b=\ell-1$ states with positive drift and $a=1$ state with negative drift. Therefore, based on the discussion in Subsection III-B and (9), one can obtain the matrices (using the method proposed by [22]) $\boldsymbol{g}, \boldsymbol{A}, \boldsymbol{H}$, and $\boldsymbol{d}$ of size $1 \times b$, $b \times b, b \times \ell$, and $1 \times \ell$, respectively, so that the joint cdf vector $\boldsymbol{F}(\boldsymbol{x})$ of the MFQ $\boldsymbol{X}(\boldsymbol{t})$ is written as

$$
\boldsymbol{F}(\boldsymbol{x})=\boldsymbol{g} e^{\boldsymbol{A} x} \boldsymbol{H}+\boldsymbol{d}, x \geq 0 .
$$

We are now ready to state the following main theorem which provides expressions for the cdfs of the AoI and PAoI processes for source-1.

Theorem 1: Consider the MFQ process $\boldsymbol{X}(\boldsymbol{t}) \sim$ $\operatorname{MFQ}(\boldsymbol{Q}, \tilde{\boldsymbol{Q}}, \boldsymbol{R})$ described in (11)-(13) with its joint cdf vector $\boldsymbol{F}(\boldsymbol{x})$ given in (14). There exist two vectors $\boldsymbol{g}_{\boldsymbol{A}}$ and $\boldsymbol{h}_{\boldsymbol{A}}$ such that the cdf of the steady-state source-1 AoI process is in the form:

$$
F_{\Delta^{(1)}}(x)=1-\boldsymbol{g}_{\boldsymbol{A}} e^{\boldsymbol{A} x} \boldsymbol{h}_{\boldsymbol{A}}, x \geq 0 .
$$

Moreover, there exist two other vectors $g_{P}$ and $\boldsymbol{h}_{P}$ such that the cdf of the steady-state source-1 PAoI process is in the form:

$$
F_{\Phi^{(1)}}(x)=1-\boldsymbol{g}_{\boldsymbol{P}} e^{\boldsymbol{A} x} \boldsymbol{h}_{\boldsymbol{P}}, x \geq 0 .
$$


Proof: Recall that the part of fluid level process in successful cycles of the MFQ $\boldsymbol{X}(\boldsymbol{t})$ spent in the states of $\mathcal{S}_{2}$ and $\mathcal{S}_{3}$ overlaps with one cycle of the AoI process; see Fig. 3. Therefore, for $x \geq 0, F_{\Delta^{(1)}}(x)$ is the limit of the following expression as $t \rightarrow \infty$ :

$$
\sum_{s \in \mathcal{S}_{2} \cup \mathcal{S}_{3}} \operatorname{Pr}\left\{X_{f}(t) \leq x, X_{m}(t)=s \mid X_{m}(t) \in \mathcal{S}_{2} \cup \mathcal{S}_{3}\right\}
$$

which yields

$$
F_{\Delta^{(1)}}(x)=\frac{\sum_{s \in \mathcal{S}_{2} \cup \mathcal{S}_{3}} F_{s}(x)}{\sum_{s \in \mathcal{S}_{2} \cup \mathcal{S}_{3}} F_{s}(\infty)}=\frac{\boldsymbol{g} e^{\boldsymbol{A} x} \boldsymbol{h}_{\boldsymbol{A}}+\boldsymbol{d} \boldsymbol{h}_{\boldsymbol{A}}^{*}}{\boldsymbol{d} \boldsymbol{h}_{\boldsymbol{A}}^{*}},
$$

where $\boldsymbol{h}_{\boldsymbol{A}}^{*}=\left[\mathbf{0}_{\boldsymbol{\ell}_{1} \times \mathbf{1}}, 1, \mathbf{1}_{\boldsymbol{\ell}_{\boldsymbol{T}}}{ }^{T}, 0\right]^{T}$ is a column vector of size $\ell$ whose entries are one corresponding to states in $\mathcal{S}_{2}$ and $\mathcal{S}_{3}$ and zero otherwise, and $\boldsymbol{h}_{\boldsymbol{A}}=\boldsymbol{H} \boldsymbol{h}_{\boldsymbol{A}}^{*}$. Note that $F_{s}(0)=0$ when $s \in \mathcal{S}_{2} \cup \mathcal{S}_{3}$ since all those states are positive drift states. Therefore, the numerator of (18) should vanish at $x=0$, i.e.,

$$
g \boldsymbol{h}_{\boldsymbol{A}}+\boldsymbol{d \boldsymbol { h } _ { A } ^ { * }}=0 .
$$

The identity (18) immediately implies (15) with the choice of $g_{A}=-g /\left(d h_{A}^{*}\right)$.

Recall also that the fluid level at the transition instance from $\mathcal{S}_{3}$ to state 4 has the same distribution as $\Phi^{(1)}$; see Fig. 3. Therefore, for $x \geq 0$, the cdf of the source-1 PAoI process denoted by $F_{\Phi^{(1)}}(x)$ can be written as the limit of the following expression as $t \rightarrow \infty$ and $\epsilon \rightarrow 0$ :

$$
\begin{aligned}
= & \operatorname{Pr}\left\{X_{f}(t) \leq x \mid X_{m}(t) \in \mathcal{S}_{3}, X_{m}(t+\epsilon)=4\right\} \\
= & \frac{\operatorname{Pr}\left\{X_{f}(t) \leq x, X_{m}(t) \in \mathcal{S}_{3}, X_{m}(t+\epsilon)=4\right\}}{\operatorname{Pr}\left\{X_{m}(t) \in \mathcal{S}_{3}, X_{m}(t+\epsilon)=4\right\}} \\
= & \frac{\sum_{j=1}^{\ell_{1}} \operatorname{Pr}\left\{X_{f}(t) \leq x, X_{m}(t)=(3,1, j), X_{m}(t+\epsilon)=4\right\}}{\sum_{j=1}^{\ell_{1}} \operatorname{Pr}\left\{X_{m}(t)=(3,1, j), X_{m}(t+\epsilon)=4\right\}} \\
= & \frac{\sum_{j=1}^{\ell_{1}}\left(\begin{array}{l}
\operatorname{Pr}\left\{X_{m}(t+\epsilon)=4 \mid X_{m}(t)=(3,1, j)\right\} \\
\operatorname{Pr}\left\{X_{f}(t) \leq x, X_{m}(t)=(3,1, j)\right\}
\end{array}\right)}{\sum_{j=1}^{\ell_{1}}\left(\begin{array}{l}
\operatorname{Pr}\left\{X_{m}(t+\epsilon)=4 \mid X_{m}(t)=(3,1, j)\right\} \\
\cdot \operatorname{Pr}\left\{X_{m}(t)=(3,1, j)\right\}
\end{array}\right.}
\end{aligned}
$$

which yields the following expression

$$
F_{\Phi^{(1)}}(x)=\frac{\sum_{j=1}^{\ell_{1}} \nu_{j}^{(1)} q_{1} F_{(3,1, j)}(x)}{\sum_{j=1}^{\ell_{1}} \nu_{j}^{(1)} q_{1} F_{(3,1, j)}(\infty)}=\frac{\boldsymbol{g} e^{\boldsymbol{A} x} \boldsymbol{h}_{\boldsymbol{P}}+\boldsymbol{d} \boldsymbol{h}_{\boldsymbol{P}}^{*}}{\boldsymbol{d} \boldsymbol{h}_{\boldsymbol{P}}^{*}},
$$

where $\boldsymbol{h}_{\boldsymbol{P}}^{*}=\left[\mathbf{0}_{\mathbf{1} \times \boldsymbol{\ell}_{1}+\mathbf{1}},\left(\boldsymbol{\nu}^{(\mathbf{1})}\right)^{T}, \mathbf{0}_{\mathbf{1} \times \boldsymbol{\ell}_{T}-\boldsymbol{\ell}_{1}+\mathbf{1}}\right]^{T}$ is a column vector of size $\ell$ whose entries are $\nu_{j}^{(1)}$ corresponding to states $(3,1, j)$, and zero otherwise, and $\boldsymbol{h}_{\boldsymbol{P}}=\boldsymbol{H} \boldsymbol{h}_{\boldsymbol{P}}^{*}$. The identity (16) follows from Eqn. (24) with the choice of $g_{P}=$ $-g /\left(d h_{P}^{*}\right)$.

To obtain the moments of the steady-state AoI, we first write the pdf of the steady-state AoI process:

$$
\begin{aligned}
f_{\Delta^{(1)}}(x) & =\frac{\mathrm{d}}{\mathrm{d} x} F_{\Delta^{(1)}}(x)=\frac{\mathrm{d}}{\mathrm{d} x}\left(1-\boldsymbol{g}_{\boldsymbol{A}} e^{\boldsymbol{A} x} \boldsymbol{h}_{\boldsymbol{A}}\right) u(x), \\
& =-\boldsymbol{g}_{\boldsymbol{A}} \boldsymbol{A} e^{\boldsymbol{A} x} \boldsymbol{h}_{\boldsymbol{A}} u(x)+\left(1-\boldsymbol{g}_{\boldsymbol{A}} \boldsymbol{h}_{\boldsymbol{A}}\right) \delta(x), \\
& =-\boldsymbol{g}_{\boldsymbol{A}} \boldsymbol{A} e^{\boldsymbol{A} x} \boldsymbol{h}_{\boldsymbol{A}} u(x)
\end{aligned}
$$

since $g_{A} h_{A}=-g h_{A} /\left(d h_{A}^{*}\right)=1$ from Eqn. (19). Therefore, $\Delta^{(1)}$ is ME-distributed (see [22] and the references therein for ME-distributions where ME stands for Matrix Exponential) and its moments can be written as:

$$
E\left[\left(\Delta^{(1)}\right)^{i}\right]=(-1)^{i} i \boldsymbol{g}_{\boldsymbol{A}} \boldsymbol{A}^{-i} \boldsymbol{h}_{\boldsymbol{A}} .
$$

One can obtain expressions for the pdf and also the moments of the random variable $\Phi^{(1)}$ using the same procedure.

In the most general case, the MFQ $\boldsymbol{X}(t)$ has a size of $\ell=\ell_{T}+\ell_{1}+2$. However, the system size can be significantly reduced in some important sub-cases. For example, consider the global preemption case, i.e., $P=\mathbf{1}_{N} \mathbf{1}_{N}^{T}$ and when the service time requirements of the sources are homogeneous, i.e., $\Theta^{(n)} \sim \Theta \sim P H(\boldsymbol{\sigma}, \boldsymbol{S})$ for $n=1, \ldots, N$ with order $\eta$ and homogeneous transmission errors and retransmission policies, i.e., $e_{n}=e, r_{n}=r, n=1, \ldots, N$. From the perspective of AoI and PAoI of the tagged source-1, one can solve an auxiliary two-source system where the second source in the auxiliary system would represent the superposition of all the sources indexed from 2 to $N$ in the original system and $P=\mathbf{1}_{\mathbf{2}} \mathbf{1}_{\mathbf{2}}^{T}$. The arising MFQ will now have an order

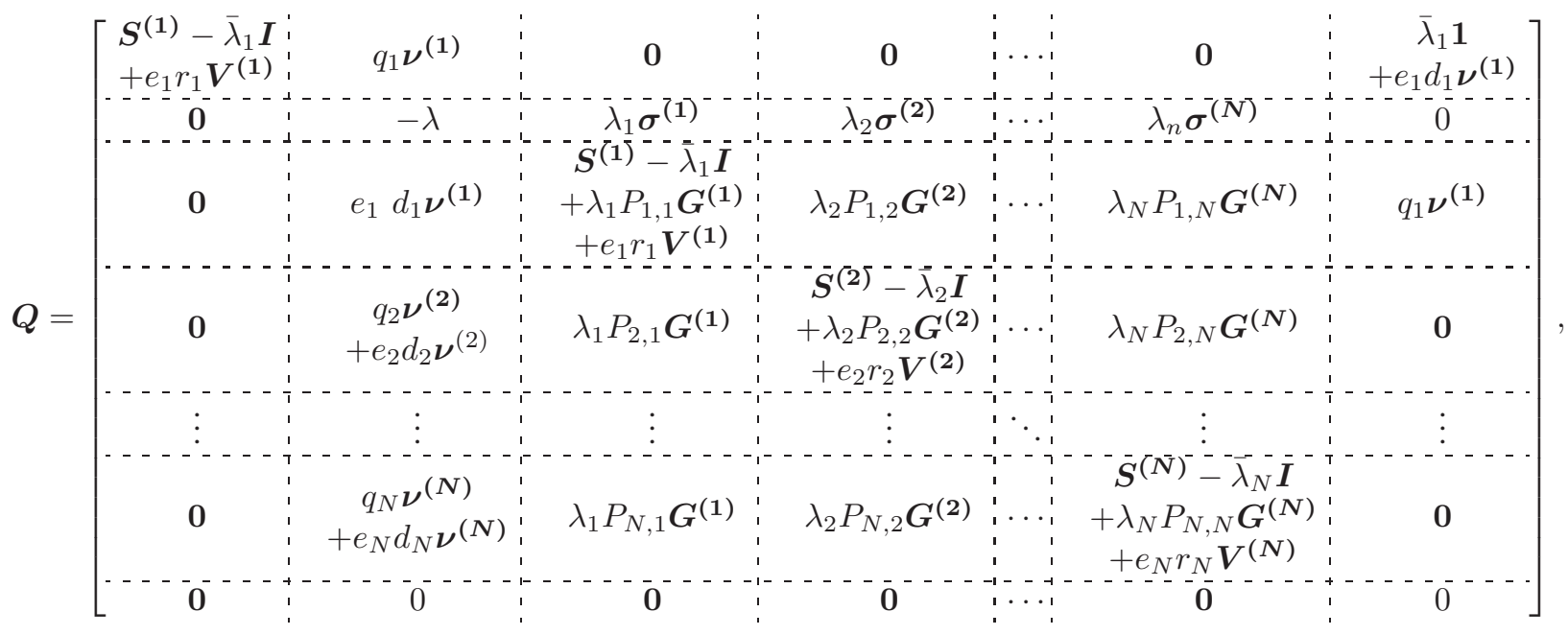


(a)

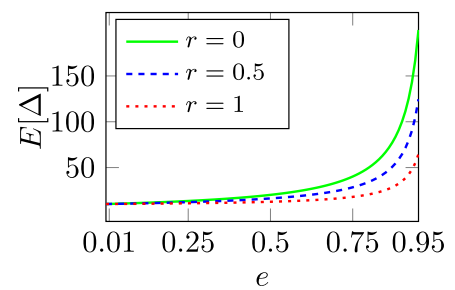

(c)
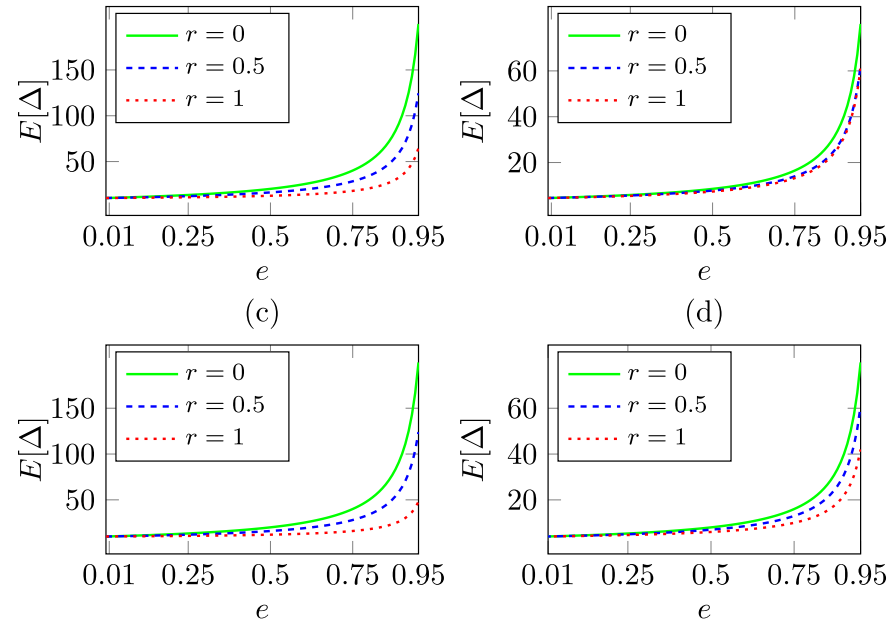

(d)

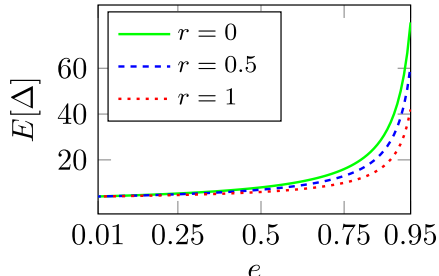

Fig. 4. The mean system AoI $E[\Delta]$ as a function of the error probability $e$ for three values of $r$ (a) NP $(\rho=0.25)$ (b) NP $(\rho=1)$ (c) GP $(\rho=0.25)$ (d) GP $(\rho=1)$.

of $3 \eta+2$ which does not depend on the number of users $N$. Similar reductions are also possible for non-preemptive systems with homogeneous service time requirements.

\section{Numerical RESUlts}

Except for one example, comparisons against simulations are not given in the current paper since not only the proposed analytical model is exact but also it relies on numerically stable and efficient vector and matrix operations. In the single example involving simulations given in Subsection V-C, the proposed model is not exact since the deterministic service time used in this example is not of PH-type but we use PH-type approximations of varying order. We refer the interested reader to [41] for comparative evaluation of the analytical model with simulations as well as the comparisons of the mean AoI and mean PAoI figures with the closed-form expressions of [8] and [28] given for certain sub-cases.

\section{A. Impact of Error and Retransmission Probabilities}

For this example, we study a two-source M/M/1/1 system with homogeneous service requirements. Particularly, we set $\mu_{n}=\mu=1, n=1,2$. The packet error probability, retransmission probability, and the traffic intensity are also assumed to be independent of the source index, i.e., $e_{n}=$ $e, r_{n}=r, \lambda_{n}=\lambda, n=1,2$. We depict in Fig. 4 the mean system AoI $E[\Delta]$ which is defined as the arithmetic average of the mean AoI values of the two sources, as a function of the error parameter $e$, for three values of the parameter $r$, and for two choices of the load parameter $\rho$ when NP and GP are deployed at the server. We observe that the mean system AoI increases with increased error probability as expected for both NP and GP policies. With GP, for a given error probability $e$, the mean system AoI appears to be larger for smaller retransmission probability $r$. For GP, we conclude that the best system AoI performance is achieved with the choice of $r=1$ with this observation being more apparent for smaller loads and for moderately lossy communications channels. The impact of $r$ is more obscured with NP at relatively higher loads since a packet which is errored is already stale since it has been generated at least one service time back. If this packet is retransmitted, it may block a potential fresh information packet arrival within its service time (more likely in high load scenarios). However, if discarded, in the case of lack of a fresh information packet arrival for an extended time (more likely in low load scenarios), the age of the source would increase. We tend to believe that the choice of $r=1$ would give rise to acceptable performance for relatively low loads for NP but at higher loads, further work needs to be carried out to derive the optimal retransmission probabilities.

\section{B. Impact of Squared Coefficient of Variation of Service Times}

In this example, we study the same homogeneous system as in the previous example but with PH-distributed service time requirements and for general $N \geq 2$. In particular, we set the homogeneous error and retransmission probabilities as $e=0.1, r=1$. For service times, we employ a PH-type distribution with mean $1 / \mu$ and squared coefficient of variation (scov) $c_{\Theta}^{2}=\sigma_{\Theta}^{2} \mu^{2}$ where $\sigma_{\Theta}^{2}$ denotes the variance of the service time. We also fix $\lambda=\mu / N$ so as to achieve a unit system load. For the purpose of constructing the PH-type distribution with given mean and scov, we use the following procedure also presented in [22]. In this procedure, the case of $c_{\Theta}^{2}=1$ leads to the exponential distribution with parameter $\mu$ for service times. When $c_{\Theta}^{2}=1 / k<1$ for a positive integer $k$, then we employ the Erlang distribution $E\left(\mu^{-1}, k\right)$ with mean $\mu^{-1}$ and order $k$ which is PH-type characterized with the matrix pair $\left(\sigma_{k}, S_{k}\right)$ where

$$
\sigma_{k}=\left[\begin{array}{llll}
1 & 0 & \cdots & 0
\end{array}\right], \boldsymbol{S}_{\boldsymbol{k}}=\mu k\left[\begin{array}{cccc}
-1 & 1 & & \\
& \ddots & \ddots & \\
& & -1 & 1 \\
& & & -1
\end{array}\right] \text {. }
$$

When $k$ is not an integer, a mixture of two Erlang distributions as given in [42] is used. When $c_{\Theta}^{2}>1$, then we use the Hyperexponential distribution with order two $\left(\mathrm{H}_{2}\right)$ with balanced means proposed in [42]. The mean residual service time of the positive random variable $\Theta$ is denoted by $m_{r}(x)=E[\Theta-x \mid \Theta>x]$ [43]. The Erlang distribution with $c_{\Theta}^{2}<1$ satisfies $m_{r}(x)<m_{r}(0)=E[\Theta]$ whereas the $\mathrm{H}_{2}$ distribution with $c_{\Theta}^{2}>1$ ensures $m_{r}(x)>E[\Theta]$ [43]. For the exponential distribution, it is clear that $m_{r}(x)=E[\Theta]$ for all $x$ due to the memoryless property. Therefore, when $c_{\Theta}^{2} \geq 1$ in our numerical examples, a fresh packet is not only more timely than the packet in service but it also does not have a longer residual service time in expectation, favoring preemption in this regime. Similarly, when $c_{\Theta}^{2}<1$ in our numerical examples, a fresh packet is more timely than the packet in service but it has a relatively longer residual service time in expectation which gives rise to a trade-off in this regime. To study the effect of $c_{\Theta}^{2}$ in more detail, we fix $\mu=1$, and depict the mean system AoI $E[\Delta]$ as a function of $c_{\Theta}^{2}$ using three different preemption policies NP, GP, and SP, for three values of $N \in\{2,4,8\}$ in Fig. 5 which reveals that 

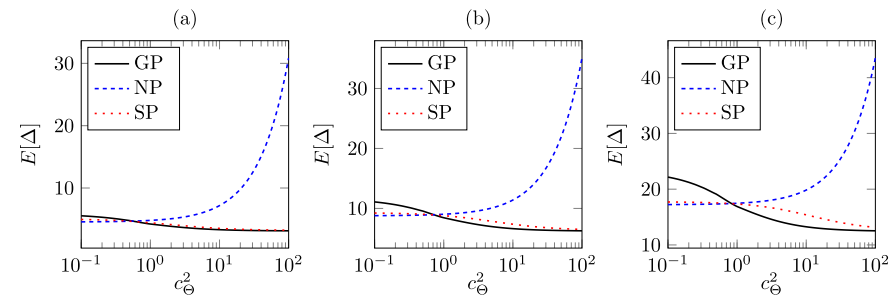

Fig. 5. The mean system AoI $E[\Delta]$ as a function of the scov parameter $c_{\Theta}^{2}$ (a) $N=2$ (b) $N=4$ (c) $N=8$. (a)

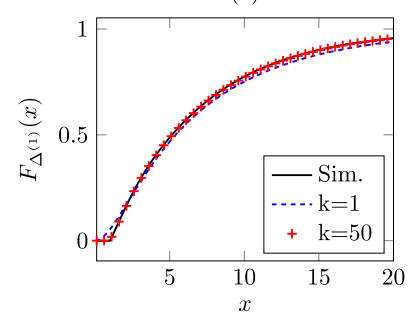

(c)

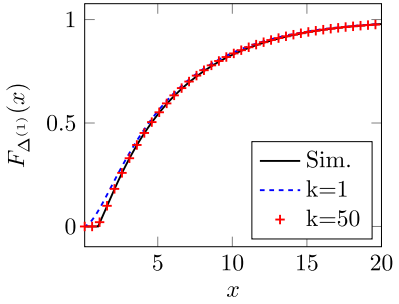

(b)

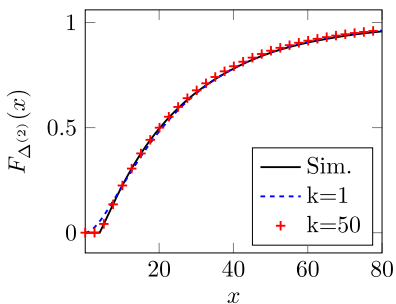

(d)

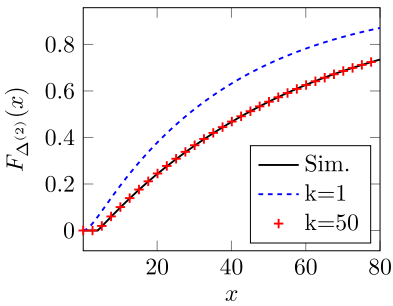

Fig. 6. The cdf of the AoI process $F_{\Delta^{(i)}}(x)$ obtained with simulations and Erlangization with order $k=1,50$ for the heterogeneous two-source $\mathrm{M} / \mathrm{PH} / 1 / 1$ system (a) NP $(i=1)$ (b) NP $(i=2)$ (c) GP $(i=1)$ (d) GP $(i=2)$.

the mean system AoI for GP and SP decreases whereas the performance of NP substantially deteriorates with increased $c_{\Theta}^{2}$. Moreover, there appears to be a threshold value for $c_{\Theta}^{2}$ above which GP outperforms NP with this threshold slightly increasing with increased $N$ but always staying below unity. Similar thresholds appear to exist above which SP outperforms NP or GP outperforms SP and all these threshold values are not necessarily identical but quite close to each other.

\section{PH-Type Approximations}

In this example, we study a two-source $\mathrm{M} / \mathrm{PH} / 1 / 1$ system with heterogeneous service time requirements both of which are deterministic and therefore service times are not PH-type. Particularly, we set $\mu_{1}=1, \mu_{2}=1 / 4, \lambda_{1}=1 / 2$, $\lambda_{2}=1 / 8$, giving rise to a heterogeneous system with $\rho_{i}=$ $1 / 2, i=1,2, \rho=1$. We employ the two Erlang distributions $E\left(\mu_{i}^{-1}, k\right), i=1,2$ of order $k$ with mean $\mu_{i}^{-1}$ to approximate the two deterministic service time distributions. This method is called Erlangization with order $k$. Note that as $k \rightarrow \infty$, the distribution $E\left(\mu^{-1}, k\right)$ converges in distribution to the deterministic distribution located at $\mu^{-1}$. Fig. 6 provides the cdf of the AoI processes (when NP and GP are employed) of both the two sources obtained with simulations and also the analytical model using Erlangization for two values of the order parameter $k=1,50$ where the $k=1$ case represents the exponential distribution with parameter $\mu$. There appears to be a perfect match with simulations when Erlangization with sufficiently high order, i.e., $k=50$, is used for all the four scenarios (a)-(d). With GP and for the second source, Erlangization with order $k=1$ produced quite poor results in terms of the match with simulations which leads us to believe that the assumption of using exponentially distributed service times matching the means only may not be a viable approach for all cases when the service times are of non-exponential nature.

\section{Impact of Choice of Preemption Probabilities}

In this subsection, we study the impact of the choice of preemption probabilities on the system cost $C(\alpha)$ for a two-source preemptive $\mathrm{M} / \mathrm{PH} / 1 / 1$ system with homogeneous service times, which is given in the following form:

$$
C(\alpha)=E\left[\Delta^{(1)}\right]+\alpha E\left[\Delta^{(2)}\right], \quad 0 \leq \alpha \leq 1,
$$

which allows one to give more importance to source-1 over source- 2 with a proper choice of the cost parameter $\alpha$. When $\alpha=1$, both sources are equally important whereas when $\alpha=0$, the age of the second source is irrelevant. For a given traffic intensity vector, load $\rho$, and $c_{\Theta}^{2}$ for the homogeneous service times, we employ a preemption matrix $\boldsymbol{P}$ such that $P_{1,1}=P_{2,2}=P_{d}$ and we perform brute-force optimization to find the optimum choices of $P_{d}, P_{1,2}$, and $P_{2,1}$, denoted by $P_{d}^{*}, P_{1,2}^{*}$, and $P_{2,1}^{*}$, respectively, so that the cost function in (29) is minimized for a given cost parameter $\alpha$ (with a resolution of 0.02 for each preemption probability parameter to be optimized). We have three different traffic mixture scenarios corresponding to the choice of the intensity vector $\lambda$ and for each mixture, the service rate $\mu$ is chosen so as to attain a unit load $\rho$. The homogeneous error and retransmission probabilities are set to 0.1 and 0.9 , respectively, for this example. Table II provides our findings. We have the following observations:

- The optimum value $P_{d}^{*}$ appears to depend on $c_{\Theta}^{2}$ and not on the specific choices of $\alpha$ and the traffic mix. When $c_{\Theta}^{2}$ is small (large), $P_{d}^{*}$ is zero (one).

- The optimum values $P_{1,2}^{*}$ and $P_{2,1}^{*}$ appear to depend on $c_{\Theta}^{2}, \alpha$, and the traffic mix. All other input parameters being fixed, $P_{2,1}^{*}$ decreases and $P_{1,2}^{*}$ increases with increased cost parameter $\alpha$ but their actual values themselves depend on the other parameters.

We also depict the cost function $C(\alpha)$ as a function of the system load $\rho$ for four preemption policies, namely non-preemptive (NP), self-preemptive (SP), globally preemptive (GP), and optimum preemptive (OP) policies (as obtained using the brute-force approach outlined above) in Fig. 7 for exponentially distributed service times, i.e., $c_{\Theta}^{2}=1$. We observe that OP significantly outperforms all the other policies with the level of performance improvement increasing with decreased cost parameter $\alpha$. When $\alpha=1$ and the traffic mix is even, global preemption and optimum preemption yield the same performance. When $\alpha=1$ and the traffic mix is not even, i.e., $\rho_{2}=2 \rho_{1}$, then there is still a substantial gain attained with the optimum preemptive policy when compared with the other preemption policies. For homogeneous exponential service times, we observe that global preemption 
TABLE II

Optimum Preemption Parameters $P_{d}^{*}, P_{1,2}^{*}$, And $P_{2,1}^{*}$, Which Minimize $C(\alpha)$ For VARIOUS VAlues OF THE TRAFFIC INTENSITY VECTOR $\boldsymbol{\lambda}, c_{\Theta}^{2}$, AND $\alpha$ WHEN $\rho=1$ AND $r_{i}=0.9$, $e_{i}=0.1, i=1,2$

\begin{tabular}{ccccccccccc}
\hline & \multicolumn{10}{c}{$\boldsymbol{\lambda}$} \\
\cline { 2 - 10 }$c_{\Theta}^{2}$ & $\alpha$ & \multicolumn{1}{c}{$[1,2]$} & \multicolumn{1}{c}{$[1,1]$} & \multicolumn{3}{c}{$[2,1]$} \\
\cline { 2 - 10 } & & $P_{d}^{*}$ & $P_{2,1}^{*}$ & $P_{1,2}^{*}$ & $P_{d}^{*}$ & $P_{2,1}^{*}$ & $P_{1,2}^{*}$ & $P_{d}^{*}$ & $P_{2,1}^{*}$ & $P_{1,2}^{*}$ \\
\hline $1 / 16$ & .25 & 0 & 1 & 0 & 0 & .68 & 0 & 0 & 0 & 0 \\
& .50 & 0 & 1 & 0 & 0 & .28 & 0 & 0 & 0 & .26 \\
& .75 & 0 & .90 & 0 & 0 & .08 & 0 & 0 & 0 & .50 \\
& 1 & 0 & .68 & 0 & 0 & 0 & 0 & 0 & 0 & .68 \\
\hline $1 / 4$ & .25 & 0 & 1 & 0 & 0 & .72 & 0 & 0 & 0 & 0 \\
& .50 & 0 & 1 & 0 & 0 & .32 & 0 & 0 & 0 & .30 \\
& .75 & 0 & .96 & 0 & 0 & .10 & 0 & 0 & 0 & .54 \\
& 1 & 0 & .74 & 0 & 0 & 0 & 0 & 0 & 0 & .74 \\
\hline 1 & .25 & 1 & 1 & 0 & 1 & 1 & .12 & 1 & 1 & 1 \\
& .50 & 1 & 1 & 0 & 1 & 1 & .54 & 1 & .58 & 1 \\
& .75 & 1 & 1 & .04 & 1 & 1 & .86 & 1 & .32 & 1 \\
& 1 & 1 & 1 & .18 & 1 & 1 & 1 & 1 & .18 & 1 \\
\hline
\end{tabular}

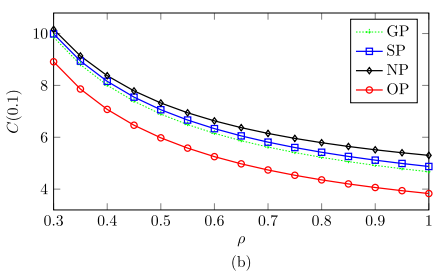

(b)

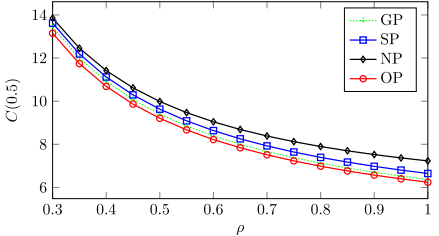

(c)

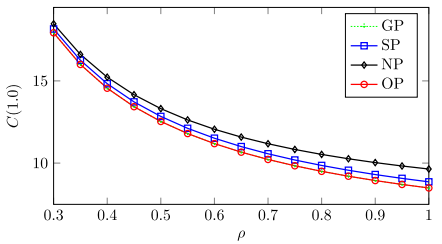

(d)

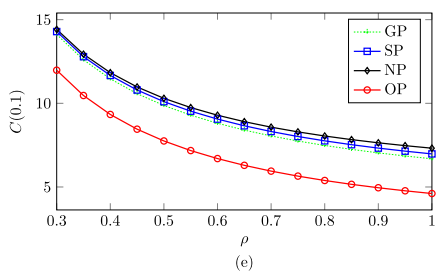

(c)

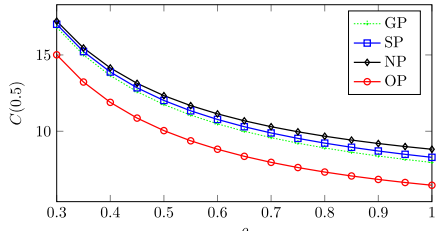

(f)

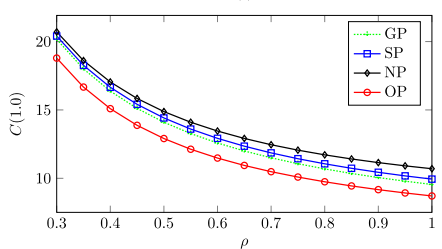

Fig. 7. The cost function $C(\alpha)$ as a function of the load parameter $\rho$ for an $\mathrm{M} / \mathrm{M} / 1 / 1$ system with four preemption policies when $\mu=1, r=0.9, e=0.1$ for three values of $\alpha \in\{0.1,0.5,1\}$. For the subfigures (a)-(c), $\rho_{1}=\rho_{2}$ whereas for the subfigures (d)-(f), $\rho_{2}=2 \rho_{1}$.

performs better than self-preemption which also outperforms the non-preemptive policy for all the cases we studied.

\section{E. Impact of Traffic Intensities}

In this subsection, we study the optimum choice of traffic intensities for a two-source M/M/1/1 system deploying an a-priori given preemption policy with homogeneous service times. We seek to minimize the total packet generation rate $\lambda_{T}=\lambda_{1}+\lambda_{2}$ that is indicative of the power needed for sensing while satisfying the following PAoI violation probabilities:

$$
\operatorname{Pr}\left\{\Phi^{(1)}>D\right\}<\varepsilon, \operatorname{Pr}\left\{\Phi^{(2)}>D\right\}<\varepsilon \alpha^{-1}, \varepsilon \leq \alpha \leq 1,
$$

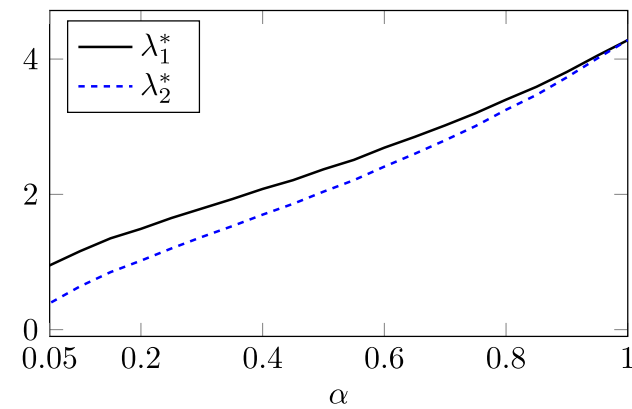

Fig. 8. The optimum source- $n$ traffic intensities $\lambda_{n}^{*}, n=1,2$ with respect to the parameter $\alpha \in[0.05,1]$.

for a violation threshold parameter $D$, violation tolerance parameter $\varepsilon$ and $\alpha$ being a parameter representing the relative importance of the second source with respect to the first; when $\alpha \rightarrow \varepsilon$, we do not have a violation constraint at all for source2 whereas when $\alpha=1$, source- 2 violation constraint is as strict as that of source-1. As an example, we set $\mu=1, D=10$, $\varepsilon=10^{-2}$ and for a given value of $\alpha$, we use the analytical model and exhaustive search to find the optimum source- $n$ traffic intensities denoted by $\lambda_{n}^{*}, n=1,2$ which are depicted in Fig. 8 as a function of the parameter $\alpha$. As we relax the violation constraint for source-2, it is possible to substantially reduce the individual traffic intensities. Moreover, as expected, the ratio $\lambda_{1}^{*} / \lambda_{2}^{*}$ increases with decreased $\alpha$.

\section{CONCLUSION}

In this paper, we propose a novel method to study the AoI and PAoI in a probabilistically preemptive bufferless heterogeneous $\mathrm{M} / \mathrm{PH} / 1 / 1$ queueing system with packet errors using Markov fluid queues. This model is more general than many recent existing AoI models arising in bufferless systems and numerically obtaining the exact distributions of AoI and PAoI is the major contribution of this paper. We show that when the service times are not PH-type, they can appropriately be approximated by PH-type distributions for the purpose of accurately obtaining the distribution of AoI. We also show through numerical examples that AoI performance attained by preemption increases with increased squared coefficient of variation of service times. When preemption is beneficial and the system cost function derived from AoI-related performance metrics is not symmetric across the sources, we show that probabilistic preemption can be tuned to outperform the conventional NP, SP, and GP policies. It is therefore concluded that probabilistic preemption policies can be used to provide source differentiation in multi-source bufferless status update systems. Future work will consist of extensions of this work to the modeling of non-preemptive status update systems with buffers and scheduling both in discrete and continuous time.

\section{REFERENCES}

[1] S. Kaul, M. Gruteser, V. Rai, and J. Kenney, "Minimizing age of information in vehicular networks," in Proc. 8th Annu. IEEE Commun. Soc. Conf. Sensor, Mesh Ad Hoc Commun. Netw., Jun. 2011, pp. 350-358.

[2] S. Kaul, R. Yates, and M. Gruteser, "Real-time status: How often should one update?" in Proc. IEEE INFOCOM, Mar. 2012, pp. 2731-2735.

[3] S. K. Kaul, R. D. Yates, and M. Gruteser, "Status updates through queues," in Proc. 46th Annu. Conf. Inf. Sci. Syst. (CISS), Mar. 2012, pp. 1-6. 
[4] N. Pappas, J. Gunnarsson, L. Kratz, M. Kountouris, and V. Angelakis, "Age of information of multiple sources with queue management," in Proc. IEEE Int. Conf. Commun. (ICC), Jun. 2015, pp. 5935-5940.

[5] A. Kosta, N. Pappas, A. Ephremides, and V. Angelakis, "Age and value of information: Non-linear age case," in Proc. ISIT, Jun. 2017, pp. 326-330.

[6] A. Kosta, N. Pappas, and V. Angelakis, "Age of information: A new concept, metric, and tool," Found. Trends Netw., vol. 12, no. 3, pp. 162-259, 2017.

[7] Y. Sun, E. Uysal-Biyikoglu, R. D. Yates, C. E. Koksal, and N. B. Shroff, "Update or wait: How to keep your data fresh," IEEE Trans. Inf. Theory, vol. 63, no. 11, pp. 7492-7508, Nov. 2017.

[8] R. D. Yates and S. K. Kaul, "The age of information: Real-time status updating by multiple sources," IEEE Trans. Inf. Theory, vol. 65, no. 3, pp. 1807-1827, Mar. 2019.

[9] Y. Inoue, H. Masuyama, T. Takine, and T. Tanaka, "A general formula for the stationary distribution of the age of information and its application to single-server queues," IEEE Trans. Inf. Theory, vol. 65, no. 12, pp. 8305-8324, Dec. 2019.

[10] M. Costa, M. Codreanu, and A. Ephremides, "On the age of information in status update systems with packet management," IEEE Trans. Inf. Theory, vol. 62, no. 4, pp. 1897-1910, Apr. 2016.

[11] K. Chen and L. Huang, "Age-of-information in the presence of error," in Proc. IEEE ISIT, Jul. 2016, pp. 2579-2583

[12] L. Huang and E. Modiano, "Optimizing age-of-information in a multiclass queueing system," in Proc. IEEE ISIT, Apr. 2015, pp. 1681-1685.

[13] A. Arafa and S. Ulukus, "Age minimization in energy harvesting communications: Energy-controlled delays," in Proc. Asilomar Conf. Signals, Syst., Comput., Oct. 2017, pp. 1801-1805.

[14] Y.-P. Hsu, E. Modiano, and L. Duan, "Age of information: Design and analysis of optimal scheduling algorithms," in Proc. IEEE Int. Symp. Inf. Theory, Jun. 2017, pp. 561-565.

[15] Q. He, D. Yuan, and A. Ephremides, "Optimal link scheduling for age minimization in wireless systems," IEEE Trans. Inf. Theory, vol. 64, no. 7, pp. 5381-5394, Jul. 2018.

[16] M. Costa, M. Codreanu, and A. Ephremides, "Age of information with packet management," in Proc. IEEE Int. Symp. Inf. Theory, Jun. 2014, pp. $1583-1587$.

[17] M. F. Neuts, Matrix-Geometric Solutions Stochastic Models: An Algorithmic Approach. New York, NY, USA: Dover, 1981.

[18] D. Anick, D. Mitra, and M. M. Sondhi, "Stochastic theory of a data-handling system with multiple sources," Bell Syst. Tech. J., vol. 61 , pp. 1871-1894, Oct. 1982.

[19] L. Kosten, "Stochastic theory of data-handling systems with groups of multiple sources," in Performance of Computer Communication Systems, H. Rudin and W. Bux, Eds. Amsterdam, The Netherlands: Elsevier, 1984, pp. 321-331.

[20] V. G. Kulkarni, "Fluid models for single buffer systems," in Frontiers in Queueing: Models and Applications in Science and Engineering, J. H. Dshalalow, Ed. Boca Raton, FL, USA: CRC Press, 1997, pp. 321-338.

21] R. D. Yates, "The age of information in networks: Moments, distributions, and sampling," IEEE Trans. Inf. Theory, vol. 66, no. 9, pp. 5712-5728, Sep. 2020.

[22] N. Akar, O. Dogan, and E. U. Atay, "Finding the exact distribution of (peak) age of information for queues of $\mathrm{PH} / \mathrm{PH} / 1 / 1$ and $\mathrm{M} / \mathrm{PH} / 1 / 2$ type," IEEE Trans. Commun., vol. 68, no. 9, pp. 5661-5672, Sep. 2020.

[23] R. D. Yates and S. Kaul, "Real-time status updating: Multiple sources," in Proc. IEEE Int. Symp. Inf. Theory (ISIT), Jul. 2012, pp. 2666-2670.

[24] Y. Inoue, H. Masuyama, T. Takine, and T. Tanaka, "The stationary distribution of the age of information in FCFS single-server queues,' in Proc. IEEE Int. Symp. Inf. Theory (ISIT), Jun. 2017, pp. 571-575.

[25] E. Najm and R. Nasser, "Age of information: The gamma awakening," in Proc. IEEE ISIT, Jul. 2016, pp. 2574-2578.

[26] A. Kosta, N. Pappas, A. Ephremides, and V. Angelakis, "Queue management for age sensitive status updates," in Proc. IEEE Int. Symp. Inf. Theory (ISIT), Jul. 2019, pp. 330-334.

[27] E. Najm and E. Telatar, "Status updates in a multi-stream M/G/1/1 preemptive queue," in Proc. IEEE Conf. Comput. Commun. Workshops (INFOCOM WKSHPS), Apr. 2018, pp. 124-129.

[28] S. Farazi, A. G. Klein, and D. R. Brown, "Average age of information in multi-source self-preemptive status update systems with packet delivery errors," in Proc. 53rd Asilomar Conf. Signals, Syst., Comput., Nov. 2019, pp. $396-400$.
[29] M. Moltafet, M. Leinonen, and M. Codreanu, "Average AoI in multi-source systems with source-aware packet management," 2020, arXiv:2001.03959. [Online]. Available: http://arxiv.org/abs/2001.03959

[30] S. K. Kaul and R. D. Yates, "Timely updates by multiple sources: The M/M/1 queue revisited," in Proc. 54th Annu. Conf. Inf. Sci. Syst. (CISS), Mar. 2020, pp. 1-6

[31] R. D. Yates, J. Zhong, and W. Zhang, "Updates with multiple service classes," in Proc. IEEE Int. Symp. Inf. Theory (ISIT), Jul. 2019 , pp. 1017-1021

[32] M. Moltafet, M. Leinonen, and M. Codreanu, "On the age of information in multi-source queueing models," IEEE Trans. Commun., vol. 68, no. 8 , pp. 5003-5017, Aug. 2020.

[33] M. Moltafet, M. Leinonen, and M. Codreanu, "Moment generating function of the AoI in multi-source systems with computationintensive status updates," 2021, arXiv:2102.01126. [Online]. Available: http://arxiv.org/abs/2102.01126

[34] N. Akar and O. Dogan, "Discrete-time queueing model of age of information with multiple information sources," IEEE Internet Things J., early access, Jan. 22, 2021, doi: 10.1109/JIOT.2021.3053768.

[35] C. A. O'Cinneide, "Characterization of phase-type distributions," Commun. Statistics. Stochastic Models, vol. 6, no. 1, pp. 1-57, Jan. 1990.

[36] S. Asmussen, O. Nerman, and M. Olsson, "Fitting phase-type distributions via the EM algorithm," Scandin. J. Statist., vol. 23, no. 4, pp. 419-441, 1996.

[37] A. Horváth and M. Telek, "PhFit: A general phase-type fitting tool," in Computer Performance Evaluation: Modelling Techniques and Tools, T. Field, P. G. Harrison, J. Bradley, and U. Harder, Eds. Berlin, Germany: Springer, 2002, pp. 82-91.

[38] H. E. Kankaya and N. Akar, "Solving multi-regime feedback fluid queues," Stochastic Models, vol. 24, pp. 425-450, Aug. 2008.

[39] A. D. S. Soares and G. Latouche, "Fluid queues with level dependent evolution," Eur. J. Oper. Res., vol. 196, no. 3, pp. 1041-1048, Aug. 2009.

[40] G. Horvath and B. Van Houdt, "A multi-layer fluid queue with boundary phase transitions and its application to the analysis of multi-type queues with general customer impatience," in Proc. 9th Int. Conf. Quant. Eval. Syst., Sep. 2012, pp. 23-32.

[41] O. Dogan and N. Akar, "The multi-source preemptive $\mathrm{M} / \mathrm{PH} / 1 / 1$ queue with packet errors: Exact distribution of the age of information and its peak," 2020, arXiv:2007.11656. [Online]. Available: http://arxiv.org/abs/2007.11656

[42] H. C. Tijms, A First Course in Stochastic Models. West Sussex, U.K. Wiley, 2003

[43] V. Iversen, Teletraffic Engineering Network Planning. Lyngby, Denmark: DTU Fotonik, 2015.

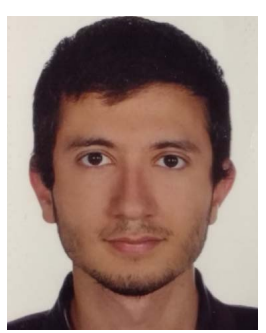

Ozancan Doğan received the B.S. and M.S. degrees in electrical and electronics engineering from Bilkent University, Ankara, Turkey, in 2018 and 2021, respectively. He is currently working as a Software Engineer at Meteksan Defence Inc., Ankara, Turkey. His current research interest includes performance modeling of age of information in communication systems.

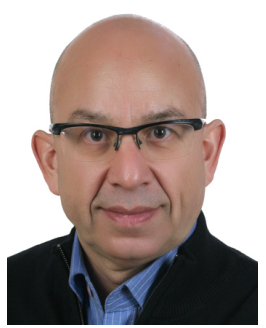

Nail Akar (Member, IEEE) received the B.S. degree from Middle East Technical University, Turkey, in 1987, and the M.S. and Ph.D. degrees from Bilkent University, Ankara, Turkey, in 1989 and 1994, respectively, all in electrical and electronics engineering. From 1994 to 1996, he was a Visiting Scholar and a Visiting Assistant Professor with the Computer Science Telecommunications Program, University of Missouri-Kansas City, USA. He joined the Technology Planning and Integration Group, Long Distance Division, Sprint, Overland Park, Kansas, in 1996, where he held a senior member of technical staff position from 1999 to 2000. Since 2000, he has been with Bilkent University, where he is currently as a Professor of the Department of Electrical and Electronics Engineering and the Associate Dean of the Faculty of Engineering. He visited the School of Computing, University of Missouri-Kansas City, as a Fulbright Scholar in 2010 for a period of six months. His research interests include performance modeling of computer and communication systems and networks, wireless networks, the Internet of Things, queueing theory, and optimization. 